\title{
OPPOSITE POLARITIES IN THE DEVELOPMENT OF SOME REGULARITIES IN THE DISTRIBUTION OF LARGE-SCALE MAGNETIC FIELDS
}

\author{
P. AMBROŽ and V. BUMBA \\ Astronomical Institute of the Czechoslovak Academy of Sciences, Ondřejov, Czechoslovakia \\ R. HOWARD \\ Hale Observatories, Carnegie Institution of Washington and \\ California Institute of Technology, Pasadena, Calif., U.S.A. \\ and \\ J. SÝYKORA \\ Astronomical Institute of the Slovak Academy of Sciences, Skalnaté Pleso, Czechoslovakia
}

\begin{abstract}
When viewed separately in the two polarities, the large-scale pattern of magnetic fields on the Sun gives an appearance reminiscent of a very large cellular structure. These structures and their relation to 'active longitudes' are discussed.
\end{abstract}

\section{Introduction}

Recently there have been several attempts to study the characteristics and motions of large-scale solar magnetic fields (Bumba and Howard, 1965a; Bumba and Howard, 1969a, b; Bumba, 1970, 1971). The suggestion was made that giant regular structures, formed from many active regions in complexes of activity, could be due to very large scale convective elements. In one study (Bumba, 1970) some indication was found that opposite polarities, either plus and minus or leading and following, behaved differently. In order to follow up this suggestion and study in more detail large-scale patterns and apparent regularities, we have undertaken this investigation, which utilizes more recent observational data which is of relatively high quality.

\section{The Observational Data}

The basic observational data are those of the Atlas of Solar Magnetic Fields 1959-1966 (Howard et al., 1967), supplemented by draft copies of magnetic synoptic charts of subsequent rotations also constructed from the daily magnetograms of the Mount Wilson Observatory. The data used extended through the end of 1969.

In addition we used synoptic charts of solar activity drawn from the Daily Maps of the Sun published by the Fraunhofer Institute in Freiburg, Germany, and the Daily Geomagnetic Character Figures $C 9$ edited by the Institut für Geophysik, Göttingen, Germany.

Because of the inhomogeneity of some of the magnetic synoptic charts and in order to cancel some rapid changes in time, the maps were integrated by overlaying two or 
three sequential maps. For example in a series of synoptic charts we would compare: $n, n+1 ; n+1, n+2 ; n+2, n+3$; etc.

\section{Two Types 'Active Longitudes'}

\section{A. 'ACTIVE LONGITUDES' ROTATINǴ WITH A 27-DAY PERIOD}

In earlier studies (Bumba and Howard, 1969a, b) it was convenient to define 'active longitudes' as the locations where large-scale patterns in the magnetic fields recurred for many rotations. Features of the background field pattern may be traced for 10 or more rotations on synoptic charts mounted in chronological order and confined to strips including only certain latitude zones. Very often individual 'rows' may be associated with either one of two individual 'streams' which may be observed to persist for several years. The inclination of such rows and streams in Carrington coordinates for latitudes between $\pm 20^{\circ}$ shows a small spread around a value corresponding to a rotation period of 27 days. A certain fine structure is noted in these inclinations (Bumba, 1971).

\section{B. 'ACTIVE LONGITUDES' ROTATING WITH A 28-DAY PERIOD}

During periods of relatively high solar activity in the equatorial strips of the synoptic charts, successive rows and streams of activity may be seen to be intensified in succession, giving the appearance on the large scale of a different rotation period. This is illustrated in Figure 1.

Another manifestation of this type of 'active longitude' is seen when comparing the equatorial strips with $20^{\circ}-40^{\circ}$ strips in either hemisphere. Individual rows are seen later displaced at higher latitudes in such a way as to give the impression of a different rotation period. This effect is best seen on the Zürich Heliographic Maps of the Photosphere which are available for four cycles of solar activity (Bumba, 1971). These synoptic charts represent photospheric faculae, which themselves represent well the distribution of magnetic field strength.

The (synodic) period represented by both effects mentioned above is close to 28 days, sometimes slightly less (Švestka, 1968), sometimes greater (Toman, 1967; Wolfer, 1897). One of us (Sykora, 1971) has recently found a 28-day rotation for 'active longitudes' formed from the green corona maximum. These results are illustrated in Figures 2 and 3. If we compare the position of Sykora's 28-day coronal 'active longitudes' with the magnetic and facular synoptic charts, we can see that the 28-day features coincide very well on both charts.

\section{The Role of 'Active Longitudes' in the Development of Large-Scale Activity}

\section{A. COMPLEXES OF ACTIVITY}

Although the boundaries of a complex of activity are in general not clearly defined, on the average the expansion during the early development of a complex proceeds at a rate of about $20 \mathrm{~m} / \mathrm{s}$ to the west and about $50 \mathrm{~m} / \mathrm{s}$ to the east. These velocities are 


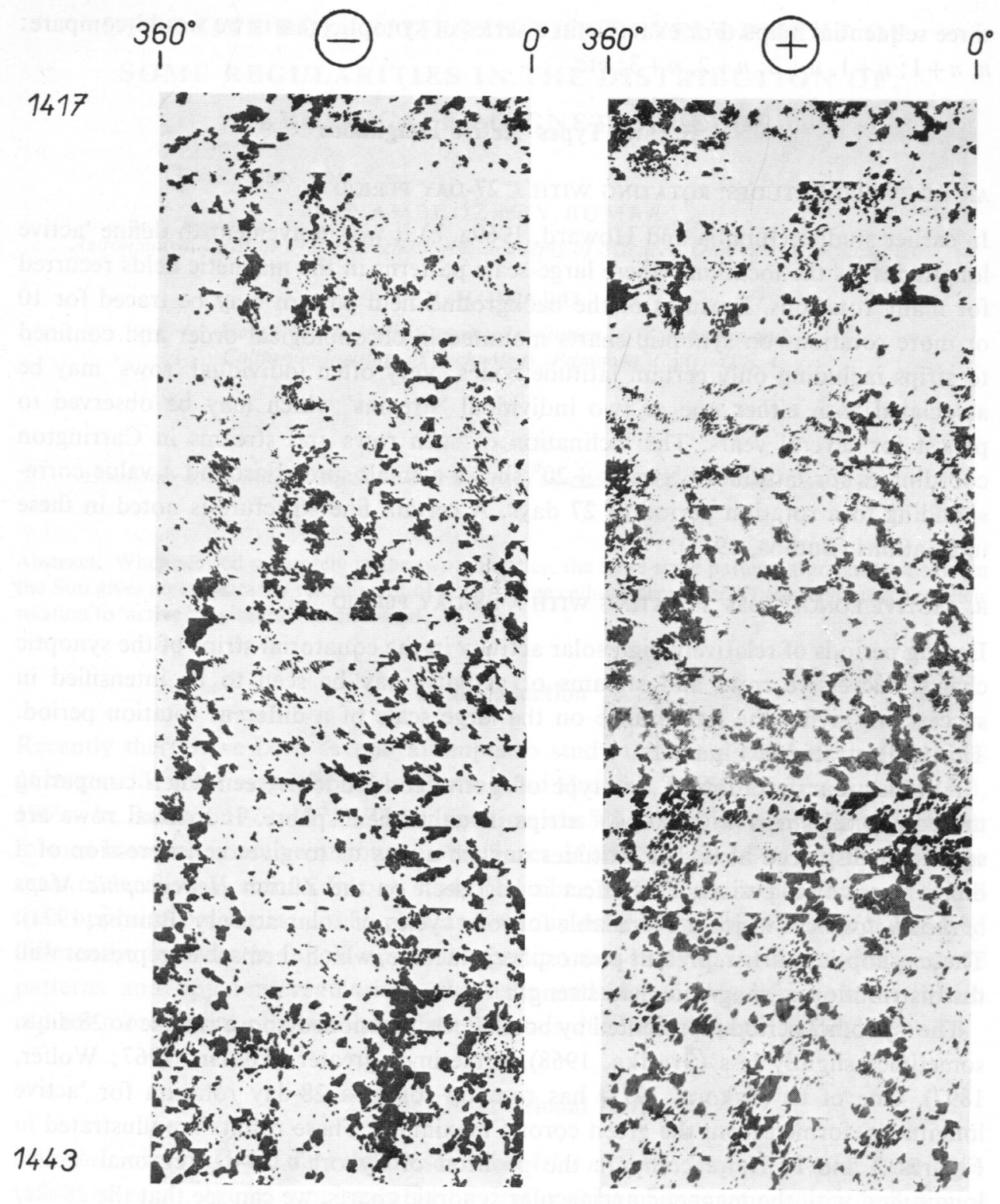

Fig. 1. Magnetic synoptic charts cut into equatorial strips $\left( \pm 20^{\circ}\right)$ and mounted in chronological order for rotations Nos. 1417-1443 (1959-1961). Both polarities are drawn separately. Minus polarity is on the left, plus on the right of the figure. The main inclination of 27 days can clearly be seen in both pictures. On the minus polarity drawing may be found the intensification of subsequent individual magnetic rows, starting in the upper left-hand corner and going in a regular succession, more inclined in the opposite direction than the 27-day rows, toward the lower right-hand corner, forming the 28-day active longitude. 

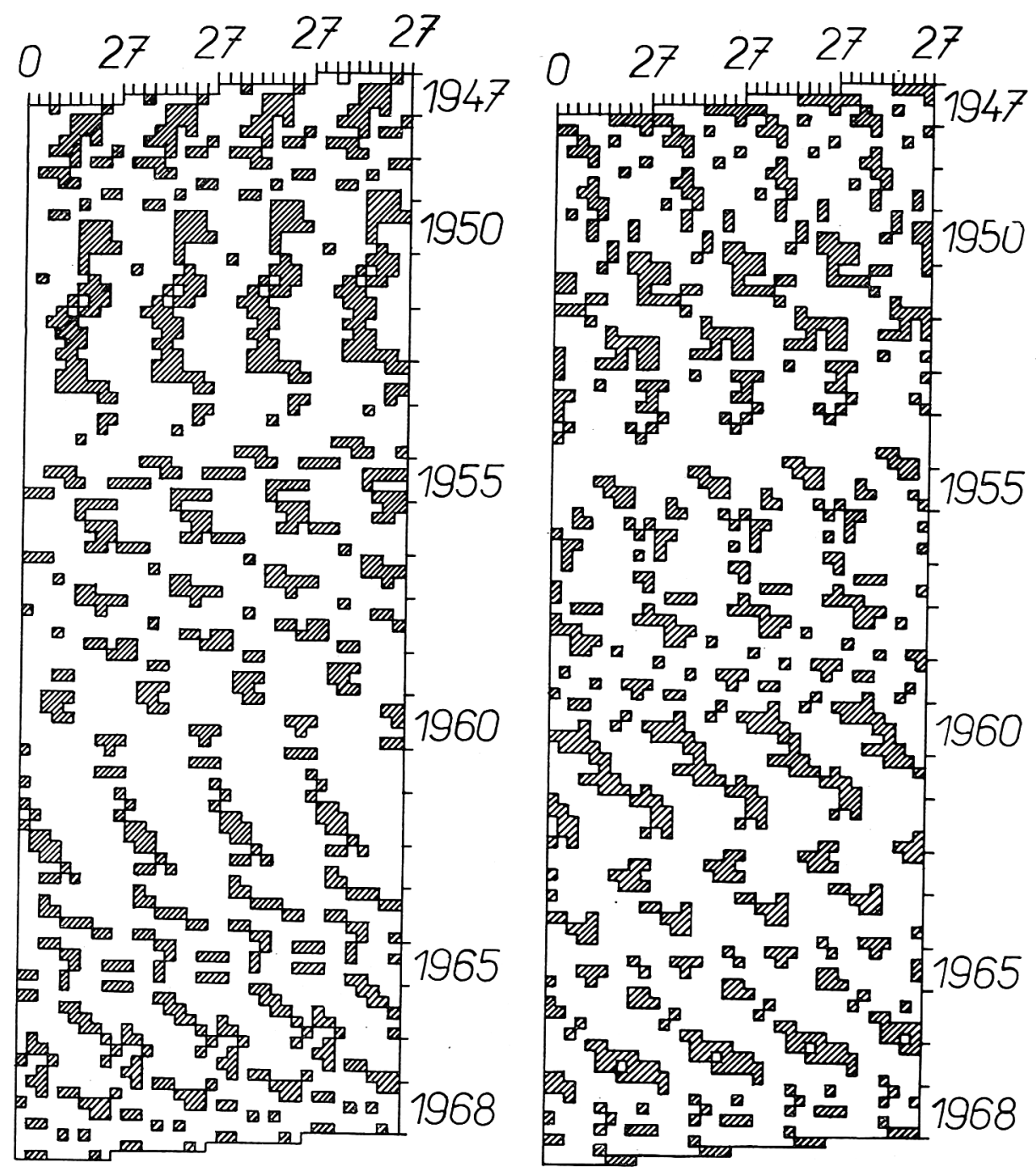

Fig. 2. Synoptic charts of green corona maxima for highest latitude intervals (to the left $+40^{\circ}-+60^{\circ}$, to the right $-40^{\circ}--60^{\circ}$ ), repeated four times, drawn in Bartel's coordinates (27-day rotation). From normalized and integrated coronal intensity for three rotations only the highest values were drawn in the form of shaded areas. During certain phases of the cycle, maxima appear with a 27-day rotation period,-during the other phase maxima with 28 -day rotational values are seen.

based on the Carrington period of 27.3 days and thus represent a period of 27 days at the west boundary and 28 days at the east boundary (Bumba et al., 1968; Sykora, 1969).

Svestka in a study of long-term forecasts of proton flares followed 81 different proton-flare regions from their appearance on the disk to their eventual decay and disappearance. He has plotted these groups in several figures. These figures show two main longitude zones of increased flare activity with interesting fine structure: there 


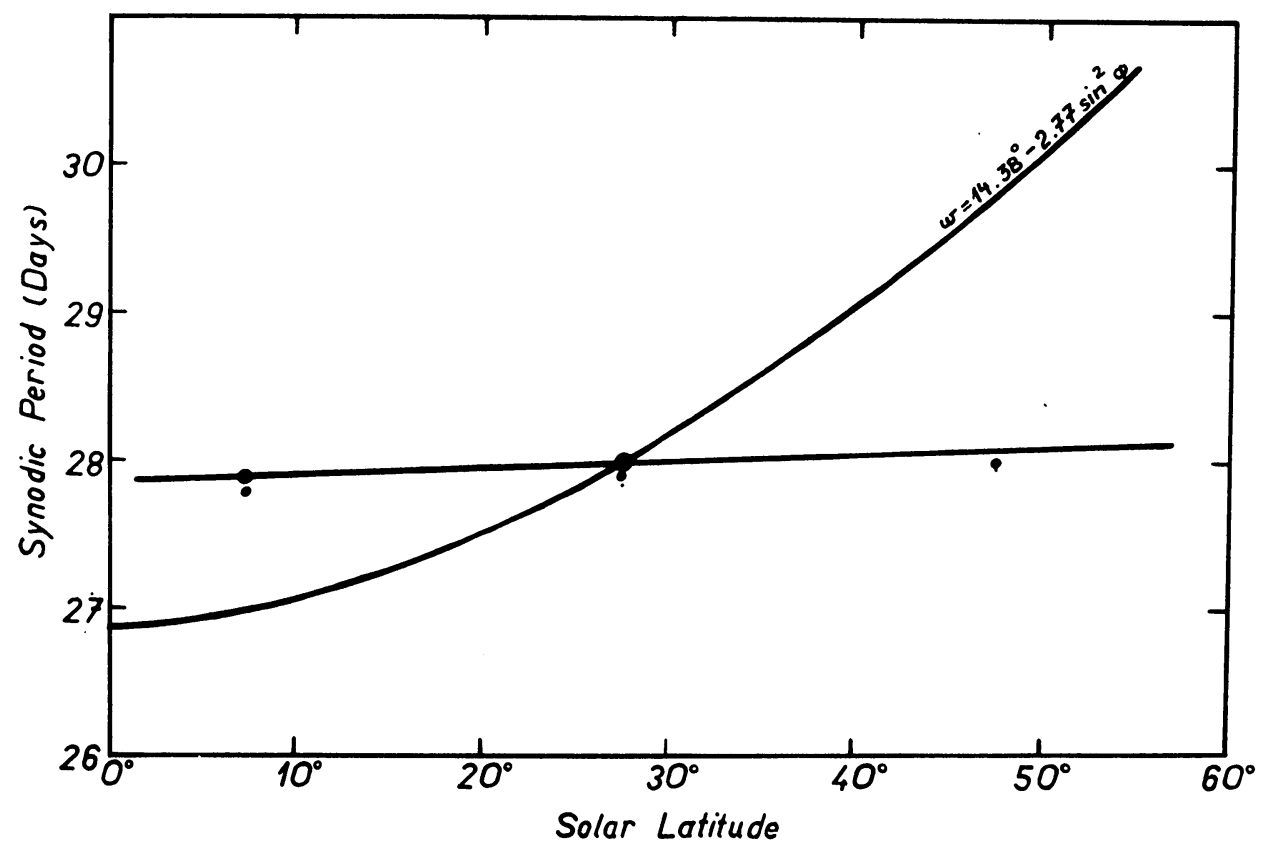

Fig. 3. The very small change of the rotational value of the 28-day active longitude in green corona in dependence on heliographic latitude (estimated for three latitude strips $\left(0-20^{\circ}, 20^{\circ}-40^{\circ}, 40^{\circ}-60^{\circ}\right)$. For comparison the differential rotation of sunspots is given.

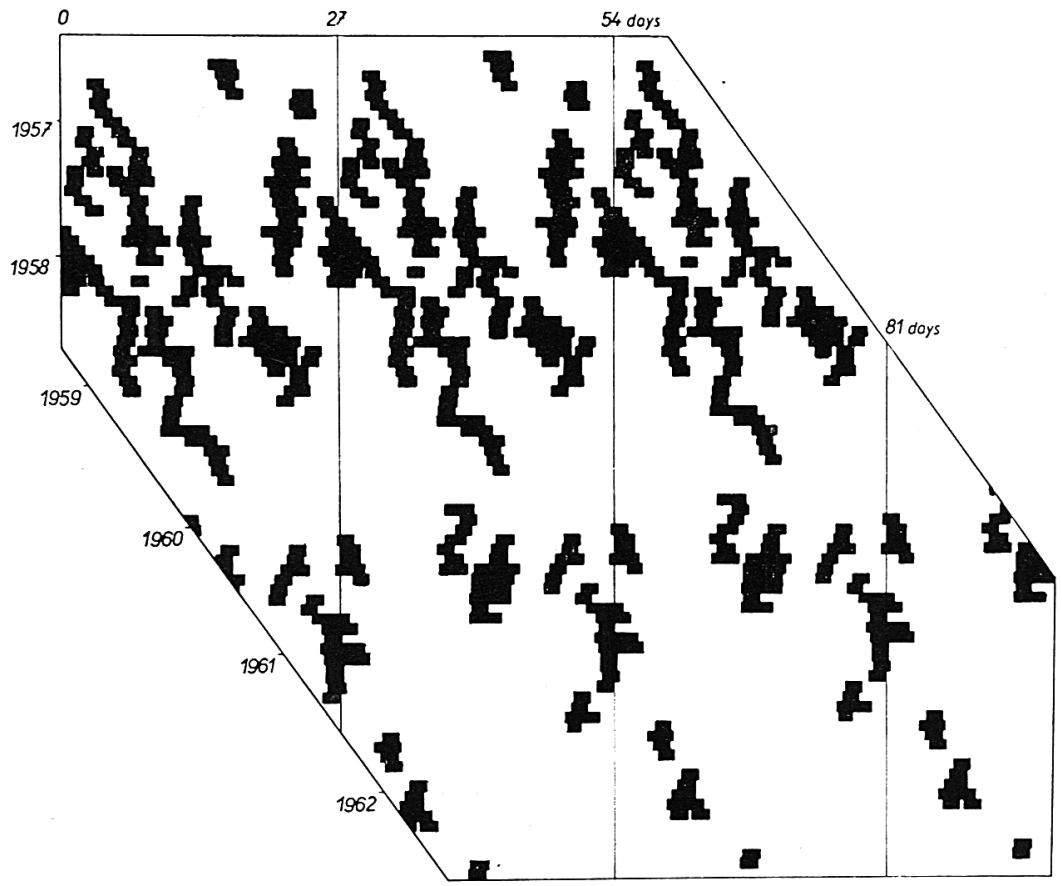

Fig. 4. Švestka's Figure 3 (1968a) showing his complexes of proton-flare activity in the southern solar hemisphere. Bartel's solar rotation periods (27 days are plotted on the horizontal axis and the subsequent solar rotation on the vertical one. The situation is repeated three times in order to stress the sequences of shorter 27-day strips forming the 28-day active longitudes. 
are individual proton-flare regions showing a 27 -day recurrence, and these relatively short-lived rows form together a longer sequence which is inclined as are the 28-day 'active longitudes'. S'vestka estimates their rotation period to be 27.8 days. See Figure 4. In the northern more active hemisphere the 27 -day features are more pronounced, and in the south the 28-day features are more clearly visible.

\section{B. 'SUPERGIANT' FEATURES}

From the definition of the 28-day 'active longitudes' it follows that the maximum in the development of both the 27-day and 28-day features occurs when these features cross on the multiple synoptic chart figure. Such maxima are formed from very large roughly elliptical structures with high latitude tails formed apparently by differential rotation. Because of the similarity of these features in appearance to granular cells, we call these 'supergiant' structures. They are best seen separately in the negative polarity magnetic fields. They are related to the development of complexes of activity, and they develop through consecutive enhancement of individual patterns of the background field as the boundary of the complex expands. Such a huge structure develops and disappears in one 27-day stream, then again in the next one (to the east), and so on as they are successively crossed by the 28 -day 'active longitude'. During the decay of one structure the following one may already be forming. In about nine rotations such 'supergiant' structures develop to their maximum size - starting about the size of an active region and expanding until the size reaches about one half of the area in the active longitudes, or even more because of the tail that extends to high latitudes $\left(55^{\circ}-60^{\circ}\right)$. This effect is illustrated in Figure 5.

'Supergiant' structures which develop at different times in different longitudes are very similar. Even the structures developed in opposite polarities may be very similar, as we shall see. They also have their own internal structure in which during the periods of best visibility we may recognize the 'giant' structures $\left(30^{\circ}-35^{\circ}\right)$ and their clusters (about $60^{\circ}$ ).

\section{The Differences Between the Polarities}

\section{A. DIFFERENCES IN THE LARGE-SCALE DISTRIBUTION}

During certain time intervals the concentration of features of each polarity into one of the two principal 27-day streams, and the clear separation of the features of both polarities may be seen. The best example of this type of large-scale configuration may be seen during rotations 1434-1443 (see Figure 6). During this interval there were two principal 27-day streams. The 28-day 'active longitude' appeared to cross one of

Fig. 5. Magnetic synoptic charts for rotations Nos. 1417-1443, constructed separately for opposite polarities. The minus polarity is on the left and the plus on the right of the figure. For integration three individual charts always overlap. Three or four sequences of minus polarity 'supergiant regular structure' development may be found. In the positions of minus polarity 'supergiant' bodies, certain maxima in the plus polarity charts can be seen. Two types of cellular features especially in the plus polarity maps may be followed: sone of them rotate with a 27-day period and some of them have a constant Carrington longitude (27.3 days). 


\section{$\ominus$}

$350^{\circ} \quad 1417,18,19$

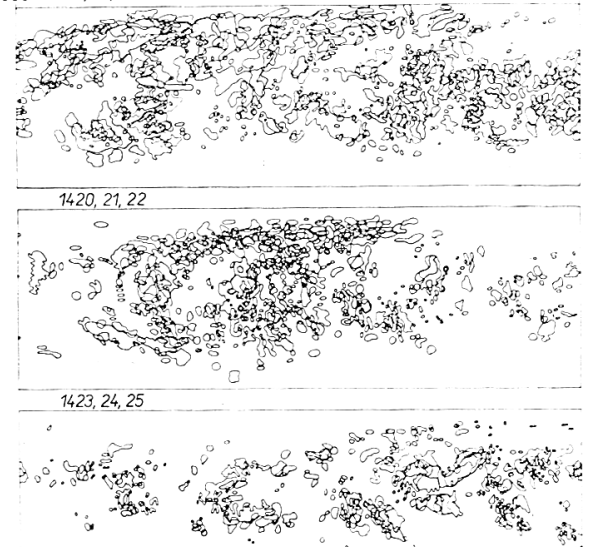

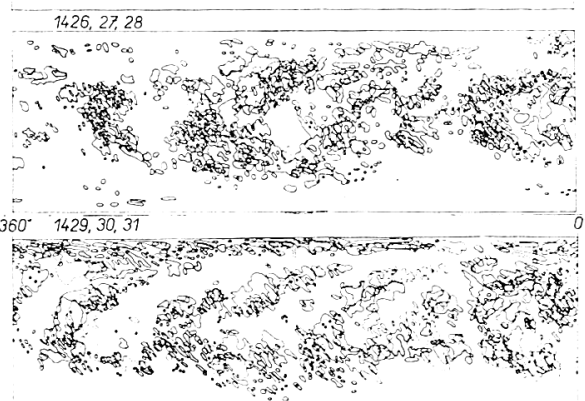

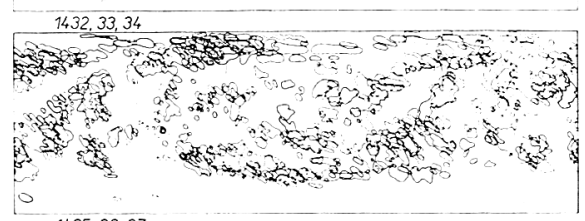

$1435,36,37$

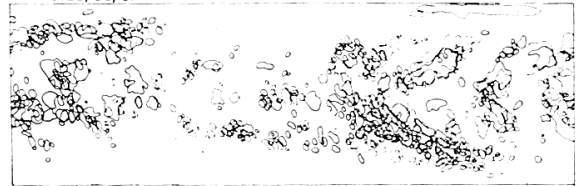
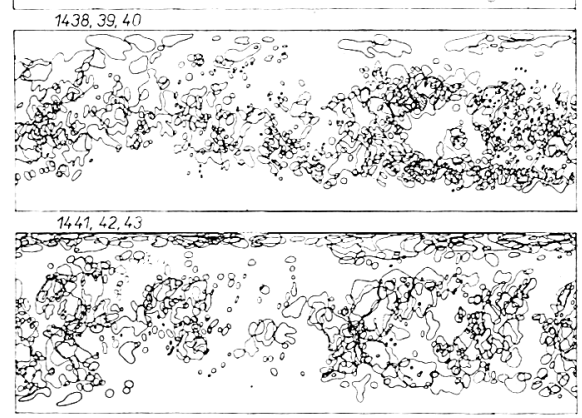

\section{$\oplus$}
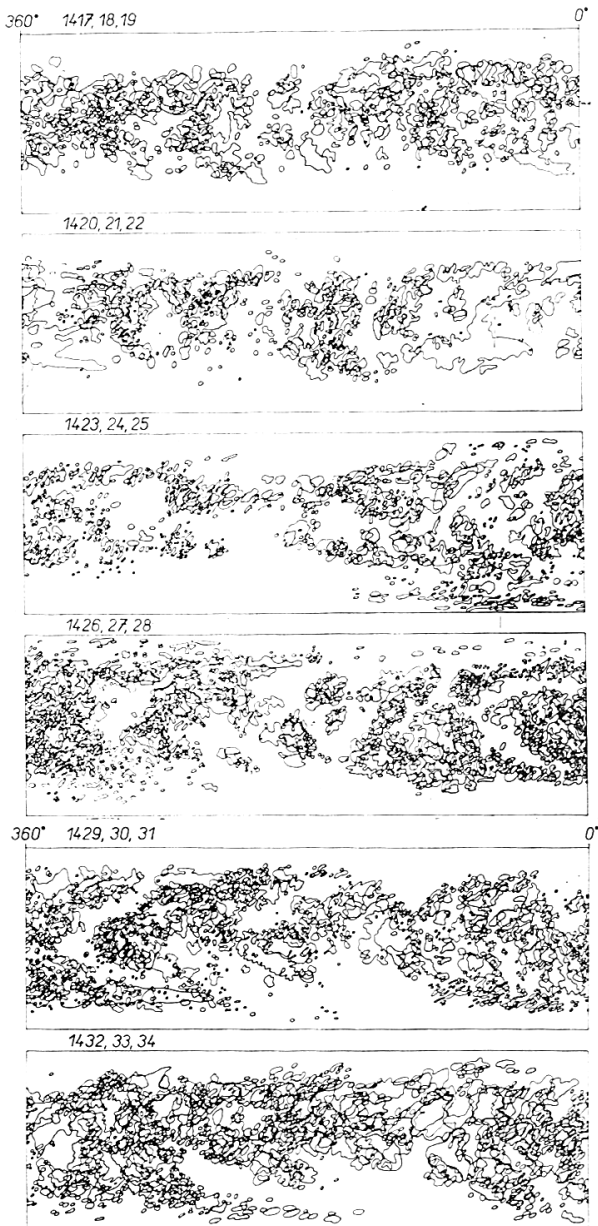

$1435,36,37$

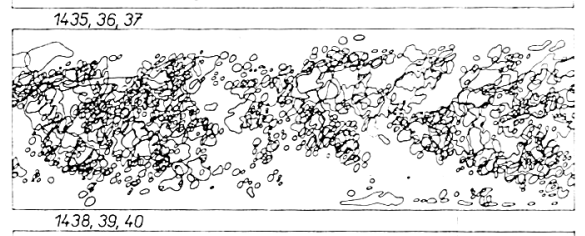

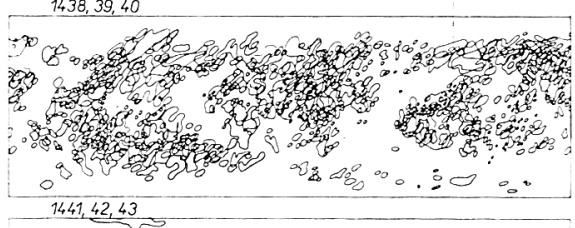

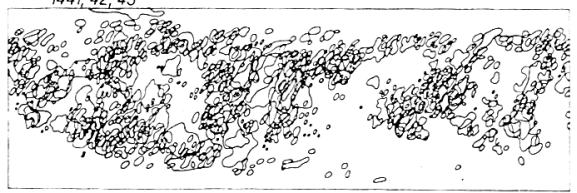

Fig. 5. 


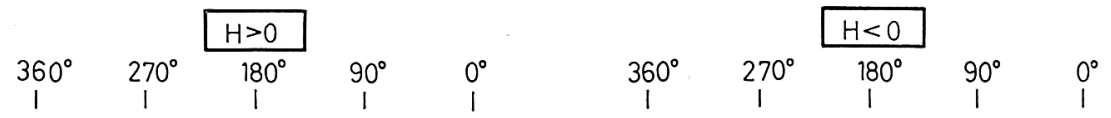

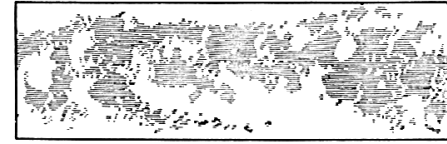

$1433-1434$

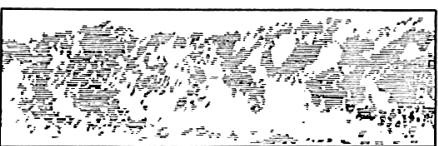

$1434-1435$

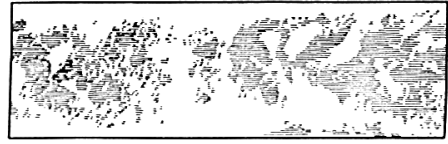

$1435-1436$

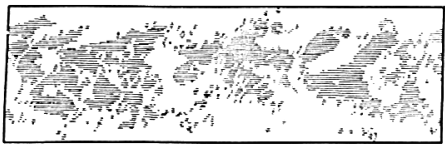

$1436-1437$

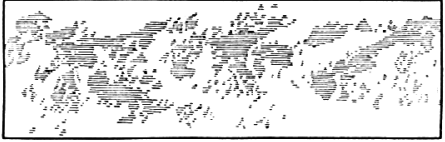

$1437-1438$

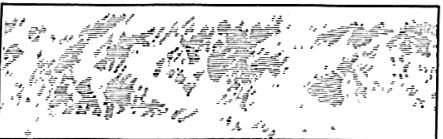

$1438-1439$

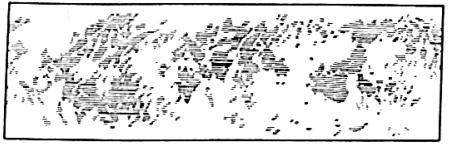

$1439-1440$

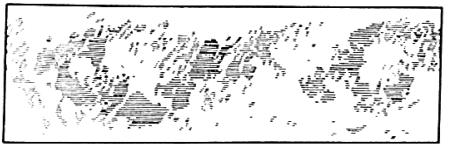

$1440-1441$

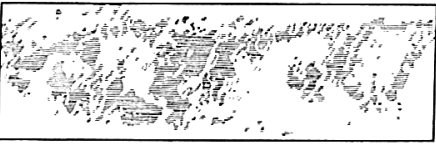

$1441-1442$

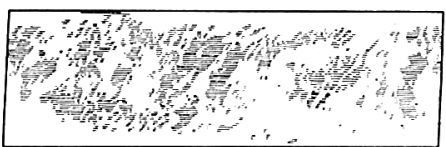

$1442-1443$

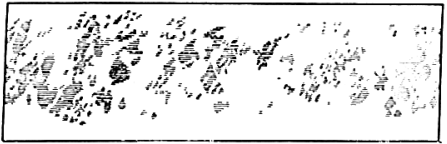

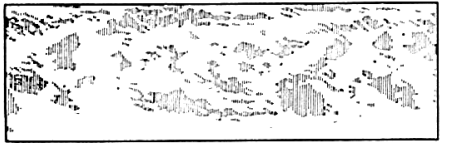
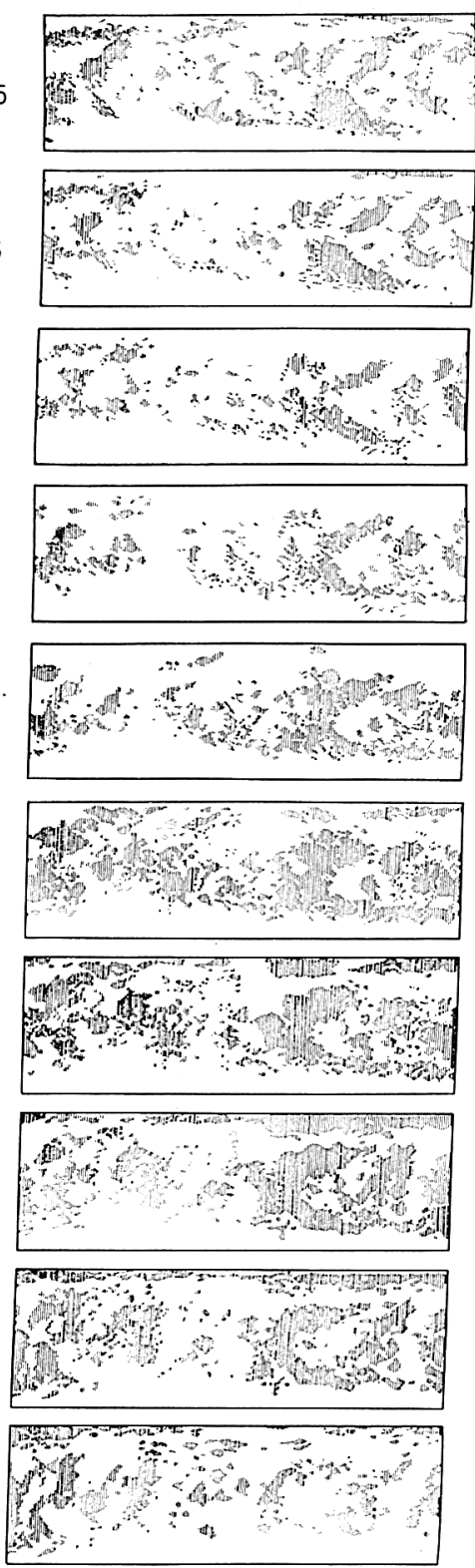

Fig. 6 . 
the streams. In this stream the negative polarity 'supergiant' structure developed, and the positive polarity 'supergiant' structure developed practically simultaneously in the other stream which was $160^{\circ}$ west of the first stream. The negative polarity feature, which was leading in the southern hemisphere, formed a tail at high southern latitudes, and the positive polarity feature formed a tail at high northern latitudes. The similarity

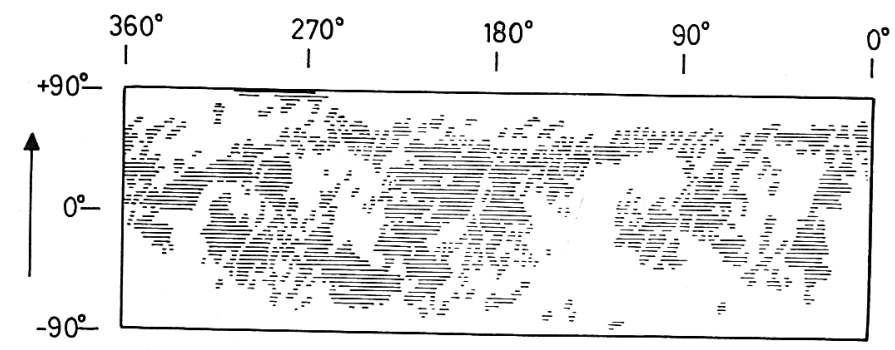

$\oplus$
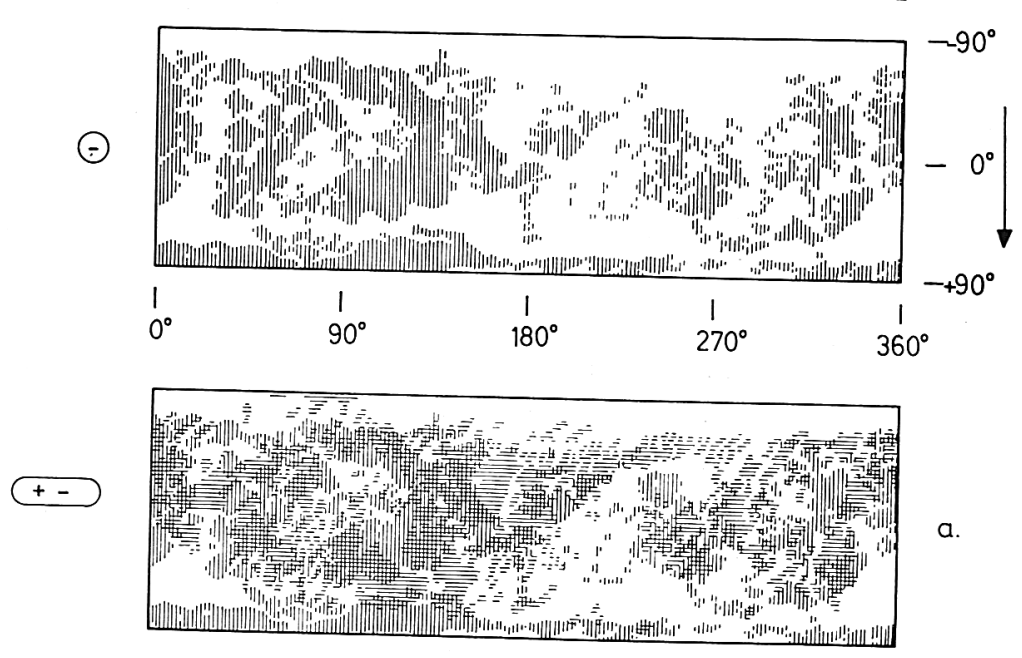

a.

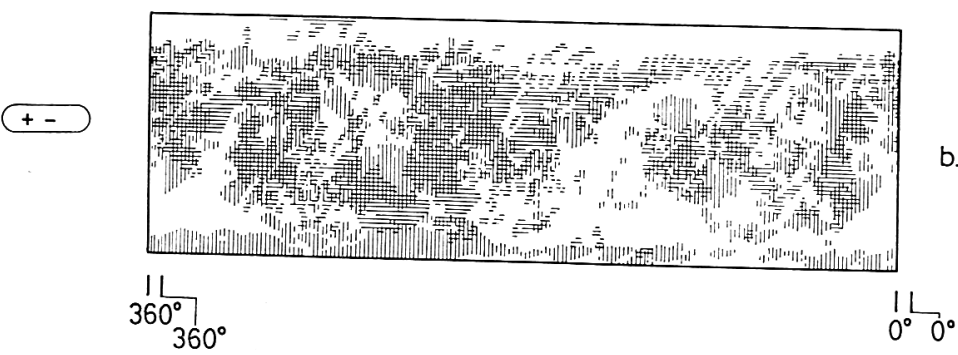

Fig. 7. Synoptic charts demonstrating the degree of symmetry of both polarity 'supergiant' structures.

Fig. 6. Magnetic synoptic charts for rotations Nos. 1434-1444 drawn in separated polarities (plus to the left, minus to the right). For integration two consecutive maps, one of which is repeated, are overlapped. The development of two 'supergiant' regular structures is shown, each of them occupying practically one half of the Sun in both main active longitudes, formed from opposite polarities. The regularity and repeated interval structure of 'supergiant' bodies and the 27-day rotational period of both active longitudes may be seen. 
of both these enormous features is so striking that for a period of time one can overlay the two and get almost perfect agreement in the position of features over nearly half the solar surface. This is illustrated in Figure 7.

On features of both polarities the characteristic structure of the 'supergiant' features may be seen during this interval. The smaller cell-like feature in the western part of the main body has a diameter of about $30^{\circ}-35^{\circ}$. The larger cell-like feature near the center of the 'supergiant' structure has a diameter of about $60^{\circ}-70^{\circ}$. The length of the whole structure is nearly $180^{\circ}$ not counting the tail.

During this time interval and viewed in this manner (polarities examined separately)
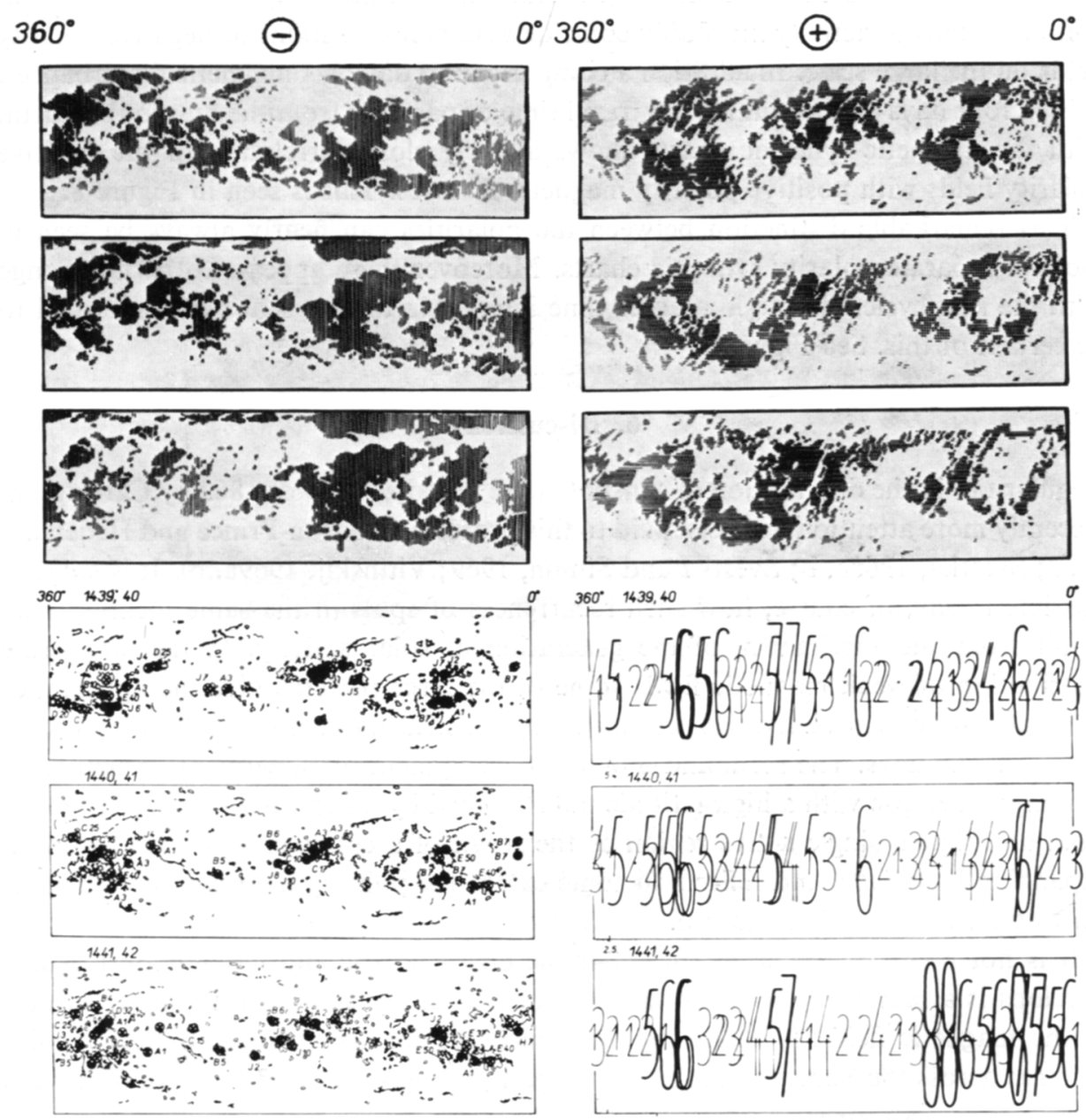

Fig. 8. Magnetic synoptic charts for rotations Nos. 1439-1442, drawn as in Figure 6, compared with the large-scale solar activity distribution (Fraunhofer Institute, Freiburg) and the geomagnetic activity distribution (Institut für Geophysik, Göttingen) for the same time interval. (The four days, needed by the particles to arrive at the Earth, are taken into account). The close relation of younger minus fields to the actual activity, as demonstrated by calcium plages, and the relation of areas of older plus fields to the geomagnetically disturbed days may be seen. 
the large-scale distribution resembles a huge bipolar sunspot group with the two polarities enhanced on either side of the Sun.

A concentration of one polarity in one stream may be observed relatively often, although the distance separating two main streams is not always $160^{\circ}$.

\section{B. CORRELATION WITH SOLAR AND GEOPHYSICAL ACTIVITY}

The frequent concentration of opposite polarity fields in streams on opposite sides of the Sun is not the only difference between the polarities. If we compare the distribution of fields of the two polarities with the distribution of activity as seen in the synoptic charts made from the Freiburg daily solar maps, we may see that concentrations of activity inevitably coincide with concentrations of negative polarity fields on the large scale. In addition a comparison of daily geomagnetic disturbances, shifted four days to account for the travel time of particles from the Sun to the Earth, with the magnetic synoptic charts shows a fairly close correlation of the positive polarity fields with positive polarity magnetic features. This is seen in Figure 8.

This remarkable distinction between the polarities can nearly always be seen in plots of separate polarity synoptic charts. Moreover there appears to be no change with the new cycle, although a larger time interval in this present cycle is needed to be certain of this. See Figure 9.

\section{Discussion}

Regularities in the distribution of sunspots were noted as early as 1863 by Carrington. Recently more attention has been paid to this problem (Dodson-Prince and Hedeman, 1968; Švestka, 1968a, b; Švestka and Simon, 1969; Vitinskij, 1969a, b). It is still not possible to say, of course, how such recurrences of spots in the same location may result from the mechanism of the generation of solar activity. In the large-scale distribution of weak magnetic fields some of the large patterns give the appearance of some sort of convective origin and rotate in 27.3 days. Some cell-like patterns rotate in $\mathbf{2 7 . 0}$ days. The form and mode of development of 'supergiant' structures is striking: an ellipse with a high-latitude tail stretched by differential rotation. It grows from an original single active region as the result of a complex of activity, with an expanding boundary, and reflects to some extent the polarity alternation of the preexisting background field pattern.

It is not now possible to say whether the characteristic enormous pattern which develops is a natural consequence of the normal redistribution on the solar surface of the magnetic fields of active regions which are formed in more or less regular patterns across the Sun, or whether the large-scale weak pattern which we observe is

Fig. 9. The same comparison of different polarity distributions with solar activity middle part for rotations Nos. 1545-1547 from the present cycle. As in the preceding cycle, the correlation of minus polarity (upper part) with solar activity may again be found. The comparison of plus polarity and geomagnetic activity distribution for this time interval was not possible because of many geomagnetic events with sudden commencements during the interval. 


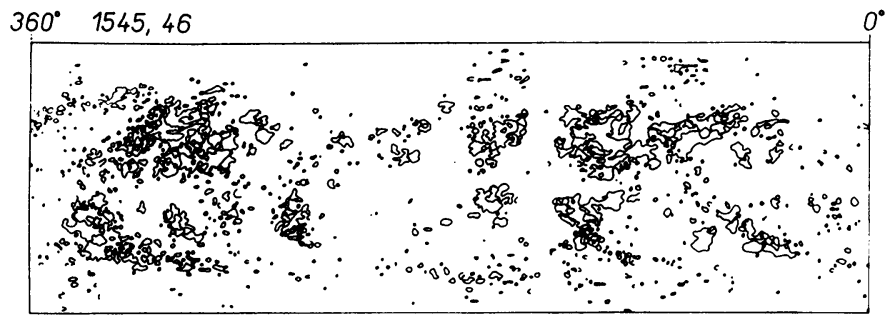

\section{6,47}
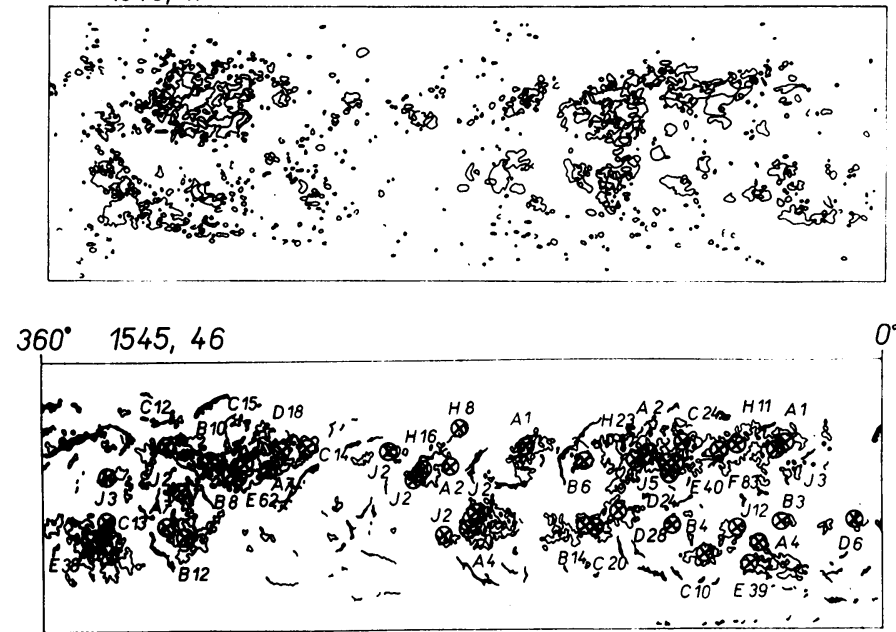

1546,47
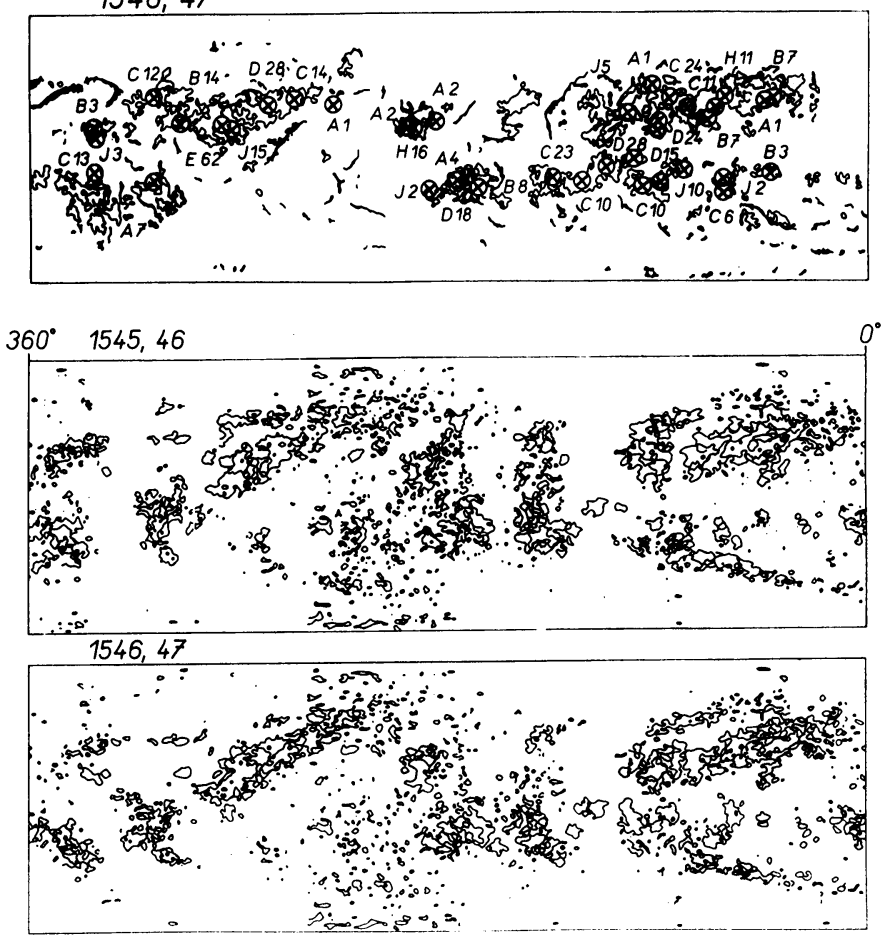

Fig. 9. 
a direct reflection of large-scale subsurface fields which themselves produce active regions at the surface in a preferential pattern.

Clearly more work is needed in the study of large-scale patterns. In particular more full-disk data of high quality are needed covering various phases of the solar cycle.

\section{Acknowledgements}

The later magnetograph observations and reductions were supported in part by the Office of Naval Research through contract No. N00014-66-C-0239.

\section{References}

Bumba, V.: 1970, Solar Phys. 11, 111.

Bumba, V.: 1971, The Proceedings of the Leningrad Symposium on Solar-Terrestrial Physics, in press.

Bumba, V. and Howard, R.: 1965a, Astrophys. J. 141, 1502.

Bumba, V. and Howard, R.: 1965b, Astrophys. J. 141, 1492.

Bumba, V. and Howard, R.: 1969a, Solar Phys. 7, 28.

Bumba, V. and Howard, R.: 1969b, in Solar Flares and Space Research (ed. by C. de Jager and Z. Svestka), North-Holland, Publ. Co., Amsterdam, p. 387.

Bumba, V., Howard, R., Martres, M. J., and Soru-Iscovici, I.: 1968, in K. O. Kiepenheuer (ed.), 'Structure and Development of Solar Active Regions', IAU Symp. 35, 13.

Carrington, R. C.: 1863, Observations of the Spots on the Sun, Williams and Norgate, London, p. 246.

Dodson-Prince, H. W. and Hedeman, E. R.: 1968, in K. O. Kiepenheuer (ed.), 'Structure and Development of Solar Active Regions', IAU Symp. 35, 56.

Howard, R., Bumba, V., and Smith, S. F.: 1967, Carnegie Inst. of Washington Publ. No. 626, Washington.

Sýkora, J.: 1969, Bull. Astron. Inst. Czech. 20, 70.

Sýkora, J.: 1971, Solar Phys. 18, 72.

Svestka, Z.: 1968a, Solar Phys. 4, 18.

Svestka, Z.: 1968b, in K. O. Kiepenheuer (ed.), 'Structure and Development of Solar Active Regions', IAU Symp. 35, 287.

Svestka, Z. and Simon, P.: 1969, Solar Phys. $10,3$.

Toman, K.: 1967, J. Geophys. Res. 72, 5570.

Vitinskij, Ju. I.: 1969a, Solar Phys. 7, 210.

Vitinskij, Ju. I.: 1969b, Soln. Dann. No. 4, 88.

Wolfer, A.: 1897, Publ. Sternw. Eidg. Polytechn. Zürich, 1, XII.

\section{Discussion}

Severny: Am I correct that you find the same effect I have just described that there is an overtaking of the south polarity by the north polarity of the general magnetic field?

Bumba: There is certainly a difference in the behavior of both polarities. The south structures change more rapidly than the north structures. In our graphs we can see also a phase shift in the maximum development of south and north structures which can make several rotations.

Severny: Did you discriminate strong fields of active centers from general magnetic fields in your analysis?

Bumba: We do not exclude the sunspot fields because the Mt Wilson magnetic maps do not see them practically, because of the large aperture $\left(17^{\prime \prime} \times 17^{\prime \prime}\right)$.

Tuominen: What is the field strength of these large scale fields?

Bumba: 4-6 G.

Tuominen: Are then these fields, the polar fields which are in the equatorial plane, more important than the 'classical' fields where the poles coincide with the poles of rotation? 
Bumba: I do not know.

Deubner: One must admit that it is very impressive to look at the large ring-like structures you showed. But could not these structures be built up from the large $\subset$ shaped flux distributions, generally present on the solar surface, together with some additional flux at the open side of these features just by chance sometimes?

Bumba: I do not think that the regularities are due to chance. They are regular not only in longitude but also in time. They repeat too often to be due to chance and you may observe their development from small to very large areas. 